\title{
ON THE POLYNOMIAL CONVERGENTS OF POWER SERIES*
}

BY W. M. WHYBURN

In this paper we will consider the power series

$$
\sum_{i=0}^{i=\infty} a_{i} z^{i}
$$

with a unit radius of convergence. M. B. Porter $\dagger$ defined a set of convergents for this series in such a way that the set had many interesting properties. $\ddagger$ He proved that at least one point of the unit circle was a limit point of the zeros of these convergents, and that in the neighborhood of every point of this circle some of the convergents took on values whose moduli were arbitrarily small. Jentzsch $\S$ has shown that every point of the unit circle is a limit point of the zeros of the complete set $\left\{f_{n}(z)\right\}$ of convergents defined by

$$
f_{n}(z)=\sum_{i=0}^{i=n} a_{i} z^{i}
$$

for all positive integral values of $n$. It is the purpose of the present paper to show that the set of convergents defined by Porter has every point of the unit circle as a limit point of its zeros. As a special case of this result we get the theorem of Jentzsch. Other properties of these convergents are developed also.

Let $f(z)$ be the analytic function defined by the above power series inside of the circle $|z|=1$ and let $z_{0}$ be a point

* Presented to the Society, April 15; 1927.

$\dagger$ Annals of Mathematics, vol. 8 (1906-7), pp. 189-192.

$\ddagger$ Of extreme importance is the fact that Porter embodied in this set of convergents all of the properties that were later assigned by Montel (see his Leģons sur les Séries de Polynomes etc., Paris, 1910) to his normal families of functions.

$\S$ Acta Mathematica; vol. 41 (1917), pp. 253-270. 
such that $\left|z_{0}\right|>1$. Let $\left\{P_{n_{i}}(z)\right\}$ be the set of convergents defined by

$$
P_{n i}(z)=a_{n i} z^{n i}\left[a_{0} z^{-n_{i}} / a_{n i}+a_{1} z^{-n_{i}+1} / a_{n i}+\cdots+1\right],
$$

where $\left|a_{n_{i}} z_{0}{ }^{n_{i}}\right|$ is greater than any of the expressions $\left|a_{j} z_{0}^{j}\right|$, for $j=0,1,2, \cdots, n_{i}-1$.

TheOREM I. Every point of the circle $|z|=1$ is a limit point of zeros of the polynomials of the set $\left\{P_{n_{i}}(z)\right\}$.

Proof. Assume the theorem false; then there exists a point $p$ on the unit circle and a circle $C^{\prime}$ with its center at $p$ such that there are no zeros of $f(z)$ or $\left\{P_{n_{i}}(z)\right\}$ within or on $C^{\prime}$. Draw the chord $x y$ within $C^{\prime}$ cutting the arc of $|z|=1$

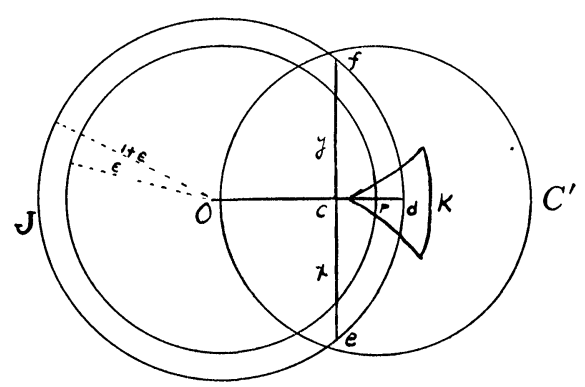

that lies within $C^{\prime}$ in such a way that the point $p$ is the midpoint of the intercepted sub-arc of this arc. Choose a positive number $\epsilon$ so that the circle $J:|z|=1+\epsilon$ cuts this chord (produced) at two points $e$ and $f$, both of which lie within $C^{\prime}$. Porter* has shown that we can pick a sub-sequence $\left\{P_{n_{j}}(z)\right\}$ of $\left\{P_{n_{i}}(z)\right\}$ such that for all $n_{j}$ greater than a fixed index $N_{1}, P_{n_{j}}(z)$ has all of its zeros except a fixed finite number, $m^{\prime}$, inside of the circle $J$. By Hurwitz' $\dagger$ theorem we can determine an index $N_{2}$ so that for all $n_{j}>N_{2}$, the $m^{\prime}$ zeros of $P_{n j}(z)$ that are not within $J$ will lie within a set $G$ of circles whose centers are at $m^{\prime}$ fixed points $\ddagger$ and whose

* Loc. cit., p. 191.

$\dagger$ Acta Mathematica, vol. 40 (1916), pp. 179-183.

$\ddagger$ These points are the zeros of the analytic function defined by $\left\{\bar{P}_{n_{j}}(z)\right\}$ outside of $J$, where $P_{n_{i}}(z)=a_{n_{i}} z^{n_{i}}\left|\bar{P}_{n_{i}}(z)^{\varphi}\right|$. 
radii are equal to a given positive constant $\epsilon^{\prime}$. We fix $\epsilon^{\prime}$ so that none of the circles of the set $G$ will have anything in common with the region $C$, where $C$ lies within $C^{\prime}$ and is bounded by the arc edf and the chord ecf ( $c$ and $d$ are the points in which the ray $O p$ cuts the chord ef and the arc ef, respectively). Since $f(c)$ is different from zero, let $|f(c)|=2 k$ and since $\lim _{n_{j} \rightarrow \infty} P_{n_{j}}(c)=f(c)$ uniformly, choose an index $N_{3}$ so that for every $n_{j}>N_{3},\left|P_{n_{j}}(c)\right|>k$.

We determine* a region $K$ that lies within $C^{\prime}$, contains $p$ and infinitely many points outside of $J$, has nothing in common with any of the circles of $G$, and such that the distance from any point of the interior of $J$ that does not belong to $C$ or the boundary of $C$ to any point of $K$ is as great as the distance from that point to $c$. Let a positive number $m$ be chosen so that the distance from any point of $K$ to any of the circles of the finite set $G$ is not less than $m$, and let another positive number $M$ be chosen so that none of the above distances are greater than $M$.

By Porter's theorem, we can find an index $N$ that is greater than $N_{1}, N_{2}, N_{3}$, such that $\left|P_{N}(s)\right|<m^{m^{\prime}} k /\left(4 M^{m^{\prime}}\right)$, where $P_{N}(z)$ belongs to $\left\{P_{n_{j}}(z)\right\}$ and $s$ is a point of $K$. Let $z_{1}$, $z_{2}, \cdots, z_{m^{\prime}}$ be the zeros of $P_{N}(z)$ that are not within $J$ and let $z_{m^{\prime}}+1, \cdots, z_{N}$ be the remaining zeros of this function;

$$
\begin{aligned}
P_{N}(z)=a_{0}\left(z-z_{1}\right)\left(z-z_{2}\right) \cdots\left(z-z_{m^{\prime}}\right) \cdots\left(z-z_{N}\right) . \\
\quad\left|P_{N}(s)\right| \\
=\left|a_{0}\right|\left|\left(s-z_{1}\right)\right| \cdots\left|\left(s-z_{m^{\prime}}\right)\right|\left|\left(s-z_{m^{\prime}+1}\right)\right| \cdots\left|\left(s-z_{N}\right)\right| \\
\geqq m^{m^{\prime}}\left|a_{0}\right|\left|\left(s-z_{m^{\prime}+1}\right)\right| \cdots\left|\left(s-z_{N}\right)\right| \\
\quad\left|P_{N}(c)\right| \leqq M^{m^{\prime}}\left|a_{0}\right|\left|\left(c-z_{m^{\prime}+1}\right)\right| \cdots\left|\left(c-z_{N}\right)\right| .
\end{aligned}
$$

Since $s$ is in $K$ and $z_{m^{\prime}+1}, \cdots, z_{N}$ are all in $J$, we have

$$
\left|\left(s-z_{m^{\prime}+1}\right)\right| \cdots\left|\left(s-z_{N}\right)\right| \geqq\left|\left(c-z_{m^{\prime}+1}\right)\right| \cdots\left|\left(c-z_{N}\right)\right|
$$

* Such a region can be constructed by using the boundaries of the set of circles through $c$ with centers within $J$ but not within $C^{\prime}$. 
and upon combining (1), (2), and (3), we get

$$
\left|P_{N}(s)\right| \geqq \frac{m^{m^{\prime}}}{M^{m^{\prime}}}\left|P_{N}(c)\right|>m^{m^{\prime}} k / M^{m^{\prime}},
$$

since $\left|P_{N}(c)\right|>k$. This contradicts the fact that $\left|P_{N}(s)\right|<$ $m^{m \prime} k /\left(4 M^{m^{\prime}}\right)$ and thus yields our theorem.

Corollary I. Every point of the circle $|z|=1$ is a limit point of the zeros of $\{f(z)\}$.

Porter* gave an example which exhibited a set of convergents that converged over a region that extended outside of the circle of convergence of the power series. This example shows that the property that we have established for our convergents does not necessarily belong to every infinite subset of $\left\{f_{n}(z)\right\}$.

In conclusion, we point out that in the neighborhood of the circle $|z|=1$ the moduli of some of the convergents of the set $\left\{P_{n_{i}}(z)\right\}$ will take on any arbitrarily assigned positive value. In this respect the circle of convergence behaves very much like an essential singularity. However, there is one point of difference in that there are no Picard numbers associated with the circle of convergence.

The University of Texas

* Loc. cit., p. 191. Ostrowski (Journal of London Society, vol. 1 (1926), part 4) has recently made an extensive study of this "over-convergence." Ostrowski gives Jentzsch credit for having called attention to this property while in reality this credit should go to Porter, whose work antedates that of Jentzsch by ten years. 